ARTICLE

\title{
Diagnostic approach to chronic kidney disease
}

\author{
I P Naiker, ${ }^{1} \mathrm{MB}$ ChB, MRCP (UK), FRCP (Lond), FCP (SA); A G Assounga, ${ }^{2} \mathrm{MD}, \mathrm{CES}, \mathrm{PhD}$; \\ A M Meyers, ${ }^{3} \mathrm{MB}$ BCh, FCP (SA), Cert Nephrology (SA), FRCP (Lond) \\ ${ }^{1}$ Private practice, St Augustine's Hospital, Durban, South Africa \\ ${ }^{2}$ Department of Nephrology, Nelson R Mandela School of Medicine, University of KwaZulu-Natal, Durban, South Africa \\ ${ }^{3}$ Donald Gordon Medical Centre, Klerksdorp Hospital, and National Kidney Foundation of South Africa, Johannesburg, South Africa
}

Corresponding author: A M Meyers (nkfsa@mweb.co.za)

Chronic kidney disease (CKD) can be considered to be present if a patient has a glomerular filtration rate $<60 \mathrm{~mL} / \mathrm{min}$ or markers of kidney disease that have been present for $>3$ months. These include proteinuria, haematuria and radiological abnormalities. Regardless of the stage of CKD, the approach is mainly similar. As stated in the South African Renal Society Guidelines for the early detection and management of $\mathrm{CKD}$, early and appropriate investigation and timeous referral of these patients enable one to establish a specific diagnosis; treat reversible diseases; optimise management to slow the progression of CKD; identify and optimally manage comorbid conditions; and plan renal replacement therapy well before the patient develops end-stage kidney disease.

S Afr Med J 2015;105(3):236. DOI:10.7196/SAMJ.9414

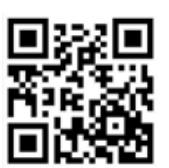

\section{Clinical aspects}

Chronic kidney disease (CKD) can be considered to be present if a patient has a glomerular filtration rate (GFR) $<60 \mathrm{~mL} / \mathrm{min}$ or markers of kidney disease that have been present for $>3$ months. These include proteinuria, haematuria and radiological abnormalities. Regardless of the stage of CKD, the approach is mainly similar. As stated in the South African Renal Society Guidelines for the early detection and management of $\mathrm{CKD}$, early and appropriate investigation and timeous referral of these patients enable one to:

- Establish a specific diagnosis and treat reversible diseases.

- Optimise management to slow the progression of CKD.

- Identify and optimally manage comorbid conditions.

- Plan renal replacement therapy well before the patient develops end-stage kidney disease.

\section{History}

As with any facet of clinical medicine, the clinician starts by taking a sound medical history. This is especially important with CKD, as the clinical manifestations are often subtle and insidious.

\section{Past history}

Patients may volunteer a history of definite kidney disease; if not, one should enquire about the following:

- a history of periorbital or peripheral oedema

- bouts of macroscopic haematuria in relation to viral infections (suggestive of immunoglobulin dimer isotype A ( $\operatorname{IgA}$ ) nephropathy)

- recurrent urinary tract infections (vesico-ureteric reflux or anatomical abnormalities)

- a past history of acute renal failure

- symptoms of prostatism.

One should enquire routinely about a past history of hypertension, diabetes, gout, renal calculi, collagen vascular disease and chronic inflammatory diseases, such as rheumatoid arthritis.

Any chronic painful condition, such as chronic headache, backache, osteoarthritis and dysmenorrhoea, may be significant, as they may result in excessive chronic analgesic intake.
- Drug history. The drugs most commonly responsible for CKD are non-steroidal anti-inflammatory drugs and compound analgesics. Remember lithium in patients with bipolar disorders. Angiotensinconverting enzyme inhibitors and angiotensin II receptor blockers tend to cause acute or subacute deterioration of renal function in patients with CKD.

- Past laboratory data. Having access to previous laboratory and radiological investigations is particularly important in $\mathrm{CKD}$, as it enables the doctor to confirm chronicity and assess the rate of progression of the disease.

- Pregnancy. A history of pregnancy-induced hypertension (in the second or third pregnancies) or early-onset pre-eclampsia suggests pre-existing hypertension or CKD.

- Family history. A family history of kidney failure or nephrotic syndrome may suggest disorders such as polycystic kidney disease, Alport syndrome, focal segmental glomerulosclerosis and IgA nephropathy. A family history of hypertension, diabetes and the metabolic syndrome assists with the evaluation of the patient's cardiovascular risk profile.

- Current symptoms. A history of anorexia, nausea, weight loss, weakness, impaired effort tolerance, nocturia and muscle cramps suggests chronicity.

\section{Physical examination}

Although the clinical features of CKD are nonspecific, the examination may reveal the following:

- General. Periorbital and/or peripheral oedema, pallor, gouty tophi, arthritis, signs of collagen vascular disease and macroglossia (amyloidosis).

- Cardiovascular. Hypertension, particularly with clinical evidence of established hypertension. Ejection systolic murmurs (anaemia) and a pericardial friction rub (uraemia).

- Chest. Pulmonary oedema suggests advanced CKD and pleural effusions occur in patients with nephrotic syndrome.

- Abdomen. Palpable kidneys (polycystic kidney disease), hepatomegaly (amyloidosis), bruits (renal artery stenosis).

- Fundoscopy. Hypertensive and diabetic retinopathy.

- Prostate gland. Hypertrophy. 
- Central nervous system. Overt confusion or more subtle impairment of intellectual functions. Generalised muscle weakness, asterixis and sensory-motor peripheral neuropathy.

\section{Special investigations}

Investigations are conducted to $(i)$ ascertain the cause of the CKD; and (ii) assess the severity and chronicity of the disease.

\section{Laboratory investigations}

- A urine examination is mandatory in all cases, i.e. dipstick, microscopy and quantitation of protein excretion. The last can be measured on a random specimen by calculating the protein/creatinine ratio. An 'active' urine sediment with microscopic haematuria and red cell casts suggests an underlying glomerulonephritis.

- Further tests to assist with the diagnosis include antinuclear antibody profile, antineutrophil cytoplasmic antibody, C-reactive protein, cryoglobulins, serum complement, hepatitis $\mathrm{B}$ and $\mathrm{C}$ profile, $\mathrm{HIV}$, venereal disease research laboratory test and uric acid levels. Serum/urine protein electrophoresis and serum Freelite assay should be done in patients $>40$ years of age with unexplained CKD and anaemia in order to exclude paraproteinaemia.

- Tests to determine the severity of the CKD and associated metabolic/haematological abnormalities include: urea and electrolytes, serum creatinine and estimated GFR. The last may be calculated by the CockcroftGault or modification of diet in renal disease (MDRD) formula or by measuring the 24-hour urinary creatinine clearance.

- Other tests include: full blood count, serum calcium, phosphate and iron studies. Alkaline phosphatase and parathyroid hormone levels are measured to assess the presence of renal osteodystrophy.

\section{Radiological investigations}

The most cost-effective examination is renal ultrasonography. This should be performed in all patients and the documentation of small echogenic kidneys supports the diagnosis of $\mathrm{CKD}$. However, the presence of normal-sized kidneys, while suggesting acute renal disease, does not exclude CKD. Ultrasound is also useful for diagnosing obstructive uropathy and may detect asymmetrical kidney size, suggesting possible renovascular disease.

Other important investigations include:

- Voiding cysto-urethrography to rule out vesico-ureteric reflux.

- A computed tomography (CT) scan (also known as the computed axial tomography
(CAT) scan) without contrast in patients with suspected renal calculi.

- Magnetic resonance angiography, CT angiography or a radio-isotope study for suspected renal artery stenosis.

In general, the clinician should - if possible - avoid exposing the patient to intravenous radio-contrast agents because of their nephrotoxicity.

\section{Kidney biopsy}

The following are indications for a kidney biopsy:

- Patients with CKD whose kidneys are normal or near normal in size, where the diagnosis cannot be made by other means.

- Patients with a definite diagnosis, where the histology is essential for appropriate management and prognosis, e.g. lupus nephritis, vasculitis.

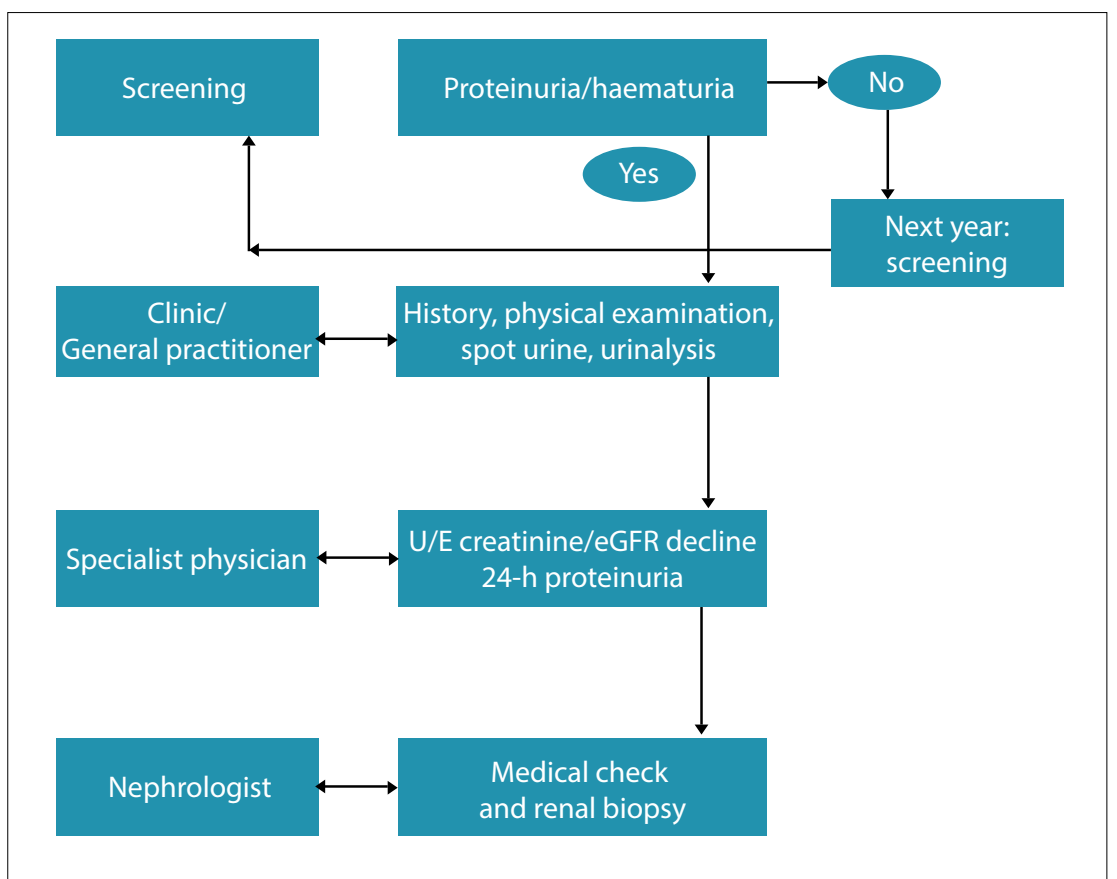

Fig. 1. Urine dipstick screening for renal disease. $(U / E=$ urea and electrolytes; $e G F R=$ estimated glomerular filtration rate.)

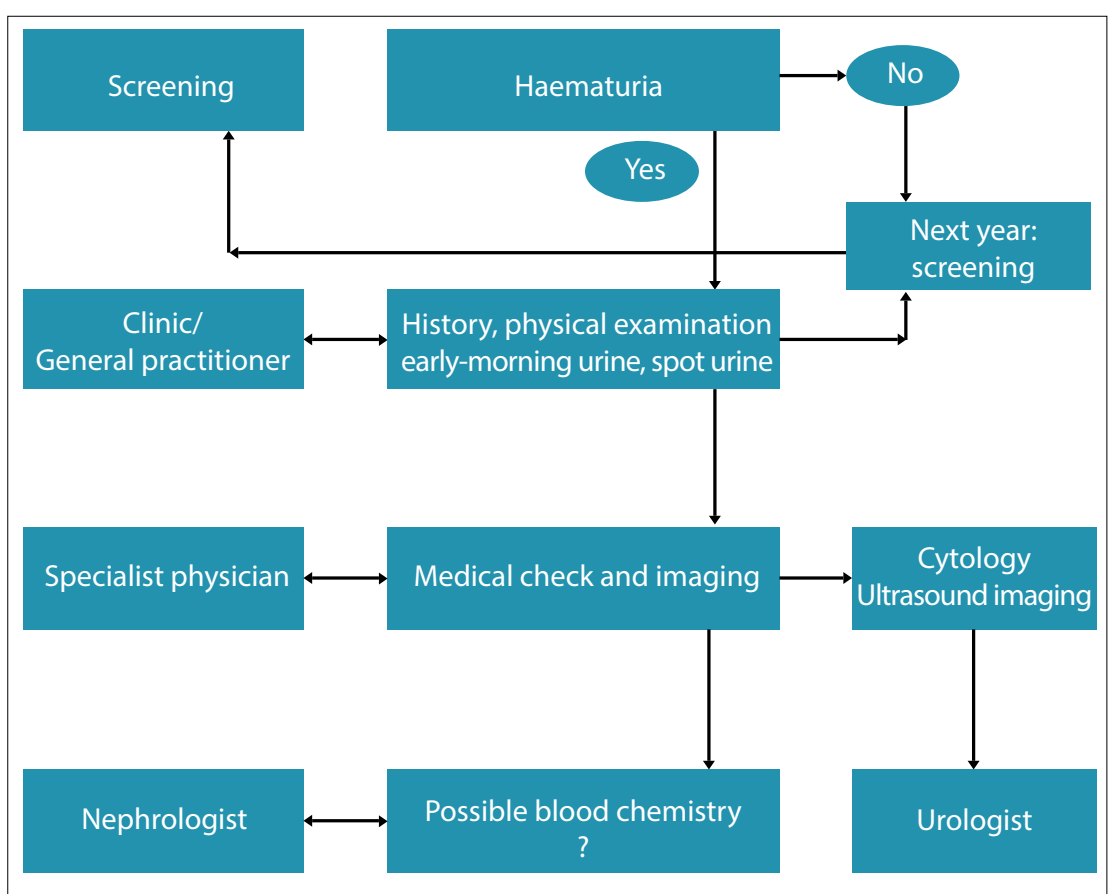

Fig. 2. Management of positive haematuria screening. 
- Patients with an established diagnosis, such as diabetic nephropathy, who have unexplained deterioration of kidney function.

Contraindications to kidney biopsy include bilateral small kidneys, uncontrolled hypertension, urinary tract or perinephric infection, polycystic kidneys and a bleeding diathesis.

\section{Significance and evaluation of urine tests}

A urine test at the bedside or in a clinic (urine dipstick) is a valuable screening and management tool.

Key findings are:

- proteinuria

- haematuria

- leucocyturia and nitrites

- other.

Proteinuria. The role of proteinuria testing using urine dipsticks, is key in the screening for renal diseases.

- A proteinuria $>3+$ is most likely of glomerular origin. Proteinuria is toxic to the tubules; hence it may lead to renal failure.

- Proteinuria of $2+$ or $1+$ is significant and needs to be further quantified to estimate the 24 -hour protein loss. This can be further quantified by measuring the protein/creatinine ratio. Timed urine collections, traditionally for 24 hours, are cumbersome and often inaccurate. These can now be replaced by the more convenient spot urine protein/creatinine ratio. A positive proteinuria test needs to be repeated to minimise the non-significant proteinuria found during heavy exercise, fever and stress.

- Common significant proteinuria is found in pyelonephritis, glomerulonephritis, nephrotic syndrome, diabetes, pre-eclampsia, HIV associated nephropathy, congestive heart failure and malignant hypertension.

Haematuria. If a urine dipstick is positive for blood, this needs to be confirmed by urine microscopy, as a urine dipstick test cannot distinguish blood from haemoglobinuria or myoglobinuria.

- Haematuria, and proteinuria, can be of renal or post-renal origin.

- Haematuria is associated with urethral causes (stricture, trauma), bladder causes (infections, tumour, trauma, stones) or ureteric (stones) renal causes, including glomerulonephritis, interstitial nephritis, tumours and trauma.

- A combination of proteinuria and haematuria may help to define the diagnosis of a condition, e.g. a diabetic patient is expected to have pure proteinura owing to diabetic nephropathy. Additional haematuria may prompt investigation for other causes, including pyelonephritis.

\section{Leucocyturia and nitrites}

- Leucocyturia often indicates infection anywhere in the urinary tract; when combined with a positive nitrite test, it is associated with infection in three-quarters of cases.

- Persistent leucocyturia could be associated with the tubercle bacillus, renal stones or interstitial nephritis.

- A urine culture needs to be performed to diagnose the causal organism, followed by an antimicrobial sensitivity test.

Other useful tests. More tests are available on some urine dipsticks; these may include $\mathrm{pH}$ and specific gravity, which are useful for the assessment of patients with acidosis and dehydration.

Figs 1 and 2 show algorithms that may assist in the management of a patient with proteinuria and/or haematuria.

\section{Essential renal function tests}

The serum creatinine - not the serum urea - is the most essential measurement. Taken alone, a mere creatinine level is not sufficient to estimate kidney function. A normal creatinine in a thin and especially elderly female, i.e. those with reduced muscle mass, could represent significant renal failure. Therefore, all patients who are at risk of CKD should have an eGFR. ${ }^{[1]}$ The previously discussed MDRD formula is more routinely available from all pathology laboratories in South Africa and laboratories express the eGFR as correlated to the definitions of CKD.

If 24-hour urine collection is to be used to estimate the GFR, there are two prerequisites: $(i)$ if the doctor explains to the patient what is required, an accurate 24-hour collection can usually be obtained, but only as an outpatient; (ii) creatinine clearance overestimates stages 1 - 4 CKD. Accurate clearance can be obtained by measuring the 24-hour urine collection (creatinine and urea clearances, adding them together and dividing by 2) (B Goldburg - personal communication). Whichever method is used, the eGFR not only stages the CKD but is essential in monitoring progress over time. eGFR tests should be performed more frequently in potentially high-risk and elderly persons.

\section{References}

1. Meyers AM. Significance, definition, classification and risk factors of chronic kidney disease in South Africa. S Afr Med J 2015;105(3):233-236. [http://dx.doi.org/10.7196/SAMJ.9412] 